\title{
An uncommon case of haemorrhagic enteric fever treated successfully with super-selective embolisation using polyvinyl alcohol particles and coils
}

Wei Yang $\underline{\mathrm{Lim}}^{1}$, MBBS, FRCR, Martin Weng Chin $\underline{H}$ 'ng $^{1}$, MBBS, FRCR, Sundeep Punamiya ${ }^{1}$, MBBS, FAMS

\begin{abstract}
Lower gastrointestinal haemorrhage due to enteric fever is uncommon and potentially fatal. The majority of patients recover with conservative treatment, with surgery reserved for life-threatening bleeding. Given the advances in radiologically guided procedures, there have been numerous reports of successful embolisation for gastrointestinal haemorrhage, although few of these involved enteric fever as the causative agent. We report an uncommon case of haemorrhagic enteric fever treated successfully with embolisation using polyvinyl alcohol particles and coils.
\end{abstract}

Keywords: catheter, embolisation, enteric fever, enteritis, haemorrhage

\section{INTRODUCTION}

Enteric fever is uncommon in Singapore but endemic in countries with poor sanitation. Gastrointestinal haemorrhage, intestinal perforation and typhoid encephalopathy are serious complications that can result from this infection. The majority of patients have mild haemorrhage that can be resolved with conservative treatment. Surgery is advocated only for lifethreatening bleeding. Advances in super-selective catheterisation and embolisation for gastrointestinal bleeding have culminated in higher treatment success rates and fewer complications. However, there is still a paucity of data regarding catheter-directed treatment for haemorrhagic enteric fever. We report one such case where haemorrhagic enteric fever was treated successfully using this method, and conclude that the method has a role to play in treatment before the condition worsens to life-threatening bleeding that necessitates surgery.

\section{CASE REPORT}

A 26-year-old Chinese woman with no noteworthy medical history was admitted to our hospital for fever, malaise, central abdominal pain and vomiting lasting more than six days. Her travel history revealed travel to Nepal two weeks prior to this admission. Clinical examination revealed mild, tender hepatomegaly. She had deranged liver function tests with raised levels of aspartate aminotransferase (278 U/L; normal range 14-41 U/L) and alanine aminotransferase (222 U/L; normal range 14-54 U/L). Hepatitis and dengue serology tests were negative, and no malaria parasites were detected on the patient's blood films. However, the patient's blood cultures grew Salmonella paratyphi A, which was sensitive to amoxicillin, and she was started on $2 \mathrm{~g}$ ampicillin six hourly.

The patient developed bloody diarrhoea four days into admission and became slightly hypotensive with a blood pressure
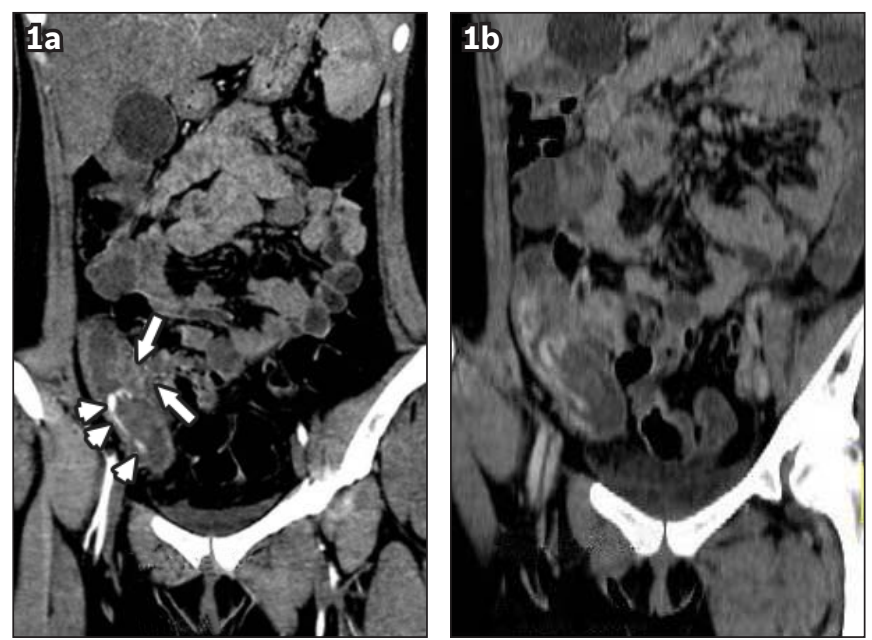

Fig. 1 (a) Early contrast-enhanced coronal CT image shows bowel wall thickening (arrows) and surrounding fat-stranding at the ileocaecal region, with active haemorrhage within the caecum (arrowheads). (b) Delayed contrast-enhanced coronal CT image shows increased pooling of contrast, indicating active haemorrhage.

of 93/59 mmHg. Her haemoglobin levels dropped from $12.8 \mathrm{~g} / \mathrm{dL}$ (normal range $11-15 \mathrm{~g} / \mathrm{dL}$ ) on admission to $6 \mathrm{~g} / \mathrm{dL}$. An urgent computed tomography (CT) angiogram showed bowel wall thickening and surrounding fat-stranding at the ileocaecal region, with active haemorrhage within the caecum (Fig. 1). Given the patient's travel history and the positive blood cultures, a diagnosis of haemorrhagic enteric enteritis was suspected. As the patient remained hypotensive despite active fluid resuscitation and blood transfusions, she was considered for emergency transcatheter embolisation. Colonoscopy was not performed, as the large amount of fresh haemorrhage was likely to obscure the bleeding site and hamper therapy.

Superior mesenteric artery angiogram via a 5F Simmons catheter (Terumo Interventional Systems, Somerset, NJ, USA) was performed, which confirmed a focus of active haemorrhage at

${ }^{1}$ Department of Diagnostic Radiology, Tan Tock Seng Hospital, Singapore

Correspondence: Dr Wei Yang Lim, Registrar, Department of Diagnostic Radiology, Tan Tock Seng Hospital, 11 Jalan Tan Tock Seng, Singapore 308433. drlimweiyang@yahoo.com 

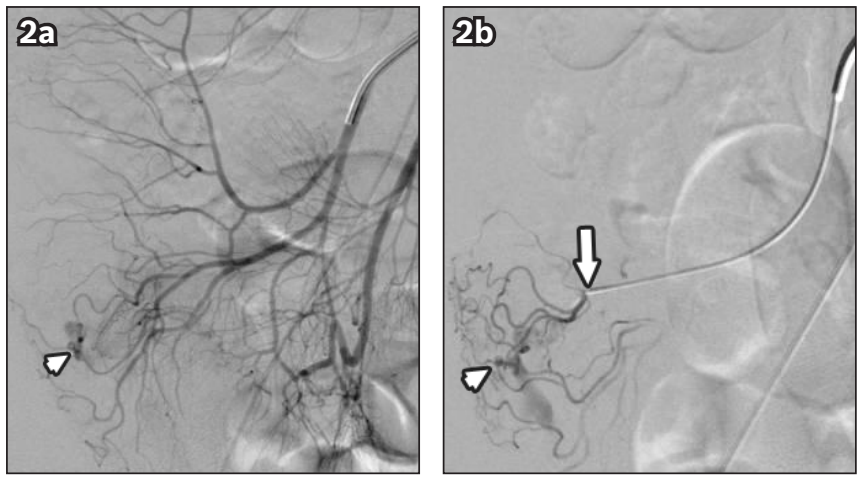

Fig. 2 (a) Angiogram, performed with the tip of the catheter within the ileocolic artery, shows a focal arterial blush in the caecum (arrowhead), confirming a focus of active haemorrhage. (b) Angiogram shows coaxial advancement of the microcatheter with the tip (arrow) at the terminal ileocolic artery. Again, focal arterial blush in the caecum (arrowhead) indicates a focus of active haemorrhage.

the caecum (Fig. 2). The catheter was advanced into the ileocolic artery and a 2.7F microcatheter (Progreat, Terumo Interventional Systems, Somerset, NJ, USA) was inserted coaxially into the bleeding vasa recta (Fig. 3). Embolisation was performed with small amounts (355-500 micron) of polyvinyl alcohol (PVA) particles (Contour, Boston Scientific Corporation, Natick, MA, USA), followed by two coils - a figure-of- 8 coil and a 4-mm complex helical coil (Boston Scientific Corporation, Natick, MA, USA). The coils were used because the PVA particles alone were unable to stop the bleeding. Post-procedure CT angiogram showed complete occlusion of the bleeding vasa recta (Fig. 4). The entire procedure was uneventful and well-tolerated by the patient.

The patient was subsequently placed in a high-dependency ward and her progress was monitored. She remained asymptomatic without any episodes of rebleeding. On Day 2 post procedure, her haemoglobin level rose to $9.7 \mathrm{~g} / \mathrm{dL}$, and on Day 5 , she was discharged with oralised cotrimoxazole to be taken 12 hourly for two weeks. At one month post procedure, the patient had a normal haemoglobin level of $11.8 \mathrm{~g} / \mathrm{dL}$ and negative stool culture. Follow-up colonoscopy was not performed, as the patient was asymptomatic.

\section{DISCUSSION}

Enteric fever is an orally transmitted systemic bacterial infection. When caused by Salmonella typhi, it is known as typhoid fever, and when due to Salmonella paratyphi A, B, or C, it is called paratyphoid fever. As both typhoid and paratyphoid fevers share similar clinical syndromes, the more inclusive term 'enteric fever' is often used.(1) Paratyphoid fever is considered milder and more infrequent than typhoid fever, and constitutes about $25 \%$ of all enteric fevers. ${ }^{(2)}$ This proportion is rapidly changing, with an increased incidence of paratyphoid being reported in countries such as Nepal; a phenomenon probably related to the fact that only vaccines protecting against Salmonella typhi are available. ${ }^{(1)}$

Enteric fever occurs primarily in developing nations with poor sanitation, and is endemic in Asia, Africa, Latin America,

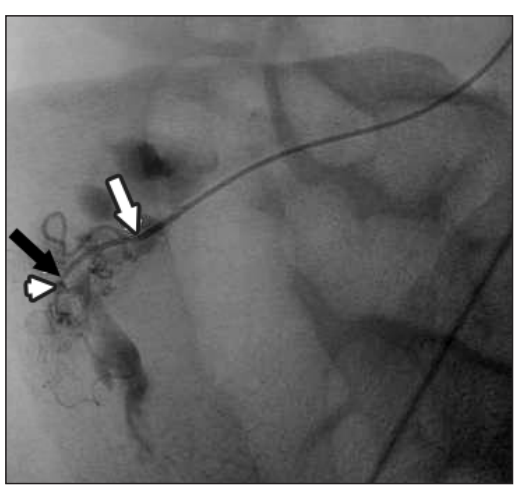

Fig. 3 Super-selective angiogram, performed with the tip of the microcatheter in the terminal ileocolic artery (white arrow), shows contrast within the vasa recta (black arrow) and further delineates the area of acute haemorrhage (arrowhead)

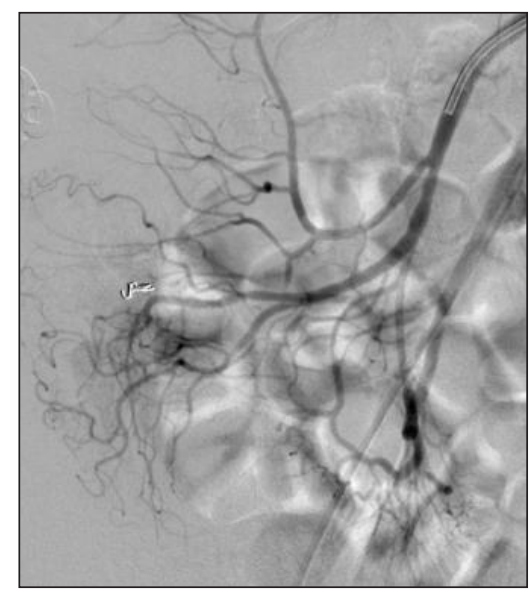

Fig. 4 Post-embolisation angiogram shows complete occlusion of the bleeding vasa recta, with no further extravasation.

the Caribbean and Oceania. It is infrequent in Singapore, with a total of 2,464 laboratory confirmed cases (1,699 cases of typhoid and 765 cases of paratyphoid) reported in the period 1990-2009. More than 90\% of cases of enteric fever in Singapore are imported, mainly from the Indian subcontinent and Indonesia. ${ }^{(3)}$ The incidence of indigenous enteric fever is low, occurring at a current annual rate of 0.26 per 100,000 population. ${ }^{(3)}$

Important complications of enteric fever include gastrointestinal haemorrhage, intestinal perforation and typhoid encephalopathy. ${ }^{(4)}$ The cause of gastrointestinal haemorrhage is the erosion of a necrotic lymphoid follicle through the wall of an enteric vessel. ${ }^{(4)}$ Bleeding is seen in up to $10 \%$ of patients, typically occurring during the third or fourth week of illness. ${ }^{(5,6)}$ In about $2 \%$ of cases, the bleeding can be severe and life-threatening, ${ }^{(4,6)}$ as in our case. Although there is scant data on the sites of bowel involvement in paratyphoid infection, ulcers in patients with typhoid fever have been found to involve the terminal ileum $(100 \%)$, ileocaecal valve (57\%), ascending colon (43\%) and transverse colon (29\%) on colonoscopy. ${ }^{(7)}$

The majority of bleeds may be treated conservatively, although the mortality rate of life-threatening bleeding is more than $30 \% .{ }^{(6)}$ While colonoscopy may be used to locate bleeding foci in enteric fever and permit adrenaline injection, mucosal fragility may render this unsafe. ${ }^{(7)}$ Furthermore, the exact site 
may be difficult to delineate if there are multiple lesions or when the site is obscured by copious haemorrhage. ${ }^{(7,8)}$ Early surgical reports have documented successful operative outcomes, and advocated surgery in the above circumstances. ${ }^{(6,9)}$ Right hemicolectomy is usually the procedure of choice.(6) Since 1968, angiography has been used to identify the bleeding point in order to aid surgical resection ${ }^{(5,10,11)}$ during this procedure, thus avoiding a blind colectomy. Angiography may also be therapeutic, ${ }^{(6,12)}$ although there is a paucity of data regarding catheter-directed treatment for haemorrhage due to enteric fever. In our review of the literature, transcatheter therapy for enteric fever bleeding using vasopressin infusion, gelfoam embolisation and coil embolisation have been described, with mixed results reported..$^{(9,11,12,13)}$

In the past, large catheter sizes with nonselective catheterisation were associated with a high rate of bowel infarction. ${ }^{(14)}$ Vasopressin has been used for lower gastrointestinal haemorrhage caused by non-typhoid sources, but intensive monitoring is required throughout administration, and treatment may be accompanied by a high incidence of rebleeding. ${ }^{(14)}$ The advent of coaxial microcatheters that permit catheterisation of the vasa recta, as well as the use of PVA particles and microcoils, have effectively reduced these adverse outcomes. ${ }^{(14)}$ Long-term follow-up of patients treated with super-selective embolisation for up to 72 months post operation has shown that the procedure should be considered as both a primary and potentially definitive treatment for life-threatening lower gastrointestinal haemorrhage. ${ }^{(15)}$ In the evaluation of lower gastrointestinal haemorrhage, CT angiography allows for rapid, noninvasive identification of areas of active bleeding, thus enabling more focused intervention. ${ }^{(14)}$ Early intervention in our patient evaded the need for open surgery and its associated morbidities. There was no evidence of rebleed on follow-up.
In conclusion, angiography offers the opportunity to be both diagnostic and therapeutic in the management of enteric fever complicated by lower gastrointestinal haemorrhage.

\section{REFERENCES}

1. Connor BA, Schwartz E. Typhoid and paratyphoid fever in travellers. Lancet Infect Dis 2005; 5:623-8.

2. Crump JA, Luby SP, Mintz ED. The global burden of typhoid fever. Bull World Health Organ. 2004; 82:346-53.

3. Ty AU, Ang GY, Ang LW, James L, Goh KT. Changing epidemiology of enteric fevers in Singapore. Ann Acad Med Singapore 2010; 39:889-96.

4. Parry CM, Hien TT, Dougan G, White NJ, Farrar JJ. Typhoid fever. N Engl J Med 2002; 347:1770-82.

5. Reyes E, Hernandez J, Gonzalez A. Typhoid colitis with massive lower gastrointestinal bleeding: an unexpected behaviour of Salmonella typhi. Dis Colon Rectum 1986; 29:511-4.

6. Bozkurt S, Celik F, Guler K. Massive lower gastrointestinal bleeding in typhoid fever. Int Surg 2004; 89:172-5.

7. Lee JH, Kim JJ, Jung JH, et al. Colonoscopic manifestations of typhoid fever with lower gastrointestinal bleeding. Dig Liver Dis 2004; 36:141-6.

8. Kuo WT, Lee DE, Saad WE, et al. Superselective microcoil embolization for the treatment of lower gastrointestinal hemorrhage. J Vasc Interv Radiol 2003; 14:1503-9.

9. Hart JL, Jackson JE. Life-threatening colonic haemorrhage in typhoid fever: successful angiographic localization and platinum microcoil embolization of several sources. Clin Radiol 2008; 63:727-30.

10. Rubin CME, Fairhurst JJ. Life-threatening haemorrhage from typhoid fever. Br J Radiol 1988; 61:415-6.

11. Suchato S, Vongvichien P, Phanchet S, Raksasook S. Diagnosis by selective arteriography in haemorrhage resulting from typhoid fever. Ann Surg 1974; 179:941-4.

12. Kearney D, Kumar A. Massive gastrointestinal haemorrhage in typhoid: successful angiographic localization and embolization. Australas Radiol 1993; 37:274-6.

13. Vaidya A, Supe A, Samsi AB, Ramakantan R. Continuous intra-arterial vasopressin infusion for control of typhoid haemorrhage. Indian J Gastroenterol 1990; 9:225-6.

14. Weldon DT, Burke SJ, Sun S, Mimura H, Golzarian J. Interventional management of lower gastrointestinal bleeding. Eur Radiol 2008; 18:857-67.

15. Ahmed TM, Cowley JB, Robinson G, et al. Long term follow-up of transcatheter coil embolotherapy for major colonic haemorrhage. Colorectal Dis 2010; 12:1013-7. 